\title{
Anesthetic experience of a patient with Prune-belly syndrome
}

\author{
Jihyun Yoon, Jaekyu Ryu, Jaewon Kim, Chang-Young Jeong, and Sun Gyoo Park \\ Department of Anesthesiology and Pain Medicine, Eulji Uneversity Medical Center, Daejeon, Korea
}

Prune-belly syndrome is a rare congenital disease $(1: 35,000-$ $1: 50,000)$ with characteristic abdominal folds that have a driedplum appearance, and the most typical triad is abdominal muscle deficiency, urinary tract abnormality, and cryptorchidism [1-4]. Other than the triad, there could be accompanying cardiovascular, musculoskeletal, gastrointestinal, respiratory, and central nerve system abnormalities in $75 \%$ of patients [2]. Due to such abnormalities, many of the patients may need diagnostic or therapeutic procedures from infancy, and general anesthesia may be required in performing procedures or operations. Patients can encounter many problems internally and surgically during anesthesia due to anatomical deficiency. Herein, the authors experienced a case in which a patient with prune-belly syndrome received anesthesia, and together with a literature review, we report several characteristics for prune-belly syndrome which anesthesiologists should take precautions for when administering anesthesia.

An 8-year-old boy weighing $20 \mathrm{~kg}$ visited the hospital for permanent catheter attachment and formulation of an $\mathrm{A}-\mathrm{V}$ fistula for hemodialysis. The patient had been diagnosed with prunebelly syndrome at birth and received an abdominoplasty when he was 3 years old. He also had a history of receiving stereotactic hematoma evacuation and closed drainage due to spontaneous intracranial hemorrhage and intraventricular hemorrhage caused by hypertension when he was 7 years old. At the time of hospitalization, the patient underwent hemodialysis three times a week for chronic renal failure and received treatment for hypertension which could not be managed well. During the hospitalization, the existing permanent catheter in the subclavian vein was removed and a femoral catheter was temporarily attached to the maintain hemodialysis.
Prune-belly patients can have accompanying renal dysfunction or renal failure from obstructive uropathy or kidney dysplasia, so renal function should be evaluated before anesthesia through BUN, creatinine, and creatinine clearance figures. In addition, any anesthesia should be carefully planned to prevent deterioration of renal function or renal failure after surgery. The patient in this case was already receiving hemodialysis treatment for chronic renal failure, so hemodialysis was to be done the day before and after the surgery.

There were no problems in mouth opening or neck extension, but intubation was expected to be difficult because the patient had a small jaw and a large tongue. Due to oligohydramnios, prune-belly patients can exhibit Potter's face with micrognathia, flattened nose, and malformed ears [1,5]. Before anesthesia, there should be preparation for circumstances where maintaining an airway is difficult through physical examination to observe symptoms of facial malformations. In addition, vomiting and regurgitation can occur frequently in prune-belly patients with uremia, so particular attention should be needed when inducing anesthesia or awakening.

The patient showed great agitation before entering the operating room so $1 \mathrm{mg}$ of midazolam was IV injected twice. After preoxygenation, anesthesia was induced with propofol $50 \mathrm{mg}$, fentanyl $20 \mu \mathrm{g}$, and rocuronium $10 \mathrm{mg}$. Manual ventilation was maintained for approximately 1 minute, and then, intubation was performed with a cuffed endotracheal tube with a $5.5 \mathrm{~mm}$ diameter. The vocal cord could be observed easily, and intubation was performed well. However, it was observed very large tongue and too small epiglottis. If vomiting occurs during anesthesia in such patients, aspiration can occur more easily than in average children, and it will be very difficult to maintain an

Corresponding author: Sun Gyoo Park, M.D., Department of Anesthesiology and Pain Medicine, Eulji Uneversity Medical Center, 95, Dunsanseo-ro, Seo-gu, Daejeon 302-799, Korea. Tel: 82-42-611-3883, Fax: 82-42-259-1111, E-mail: sgpark0626@cau.ac.kr

(c) This is an open-access article distributed under the terms of the Creative Commons Attribution Non-Commercial License (http:// creativecommons.org/licenses/by-nc/3.0/), which permits unrestricted non-commercial use, distribution, and reproduction in any medium, provided the original work is properly cited. 
airway.

Intubation can be performed in a state of spontaneous respiration without muscle relaxant, or with a minimum amount of muscle relaxant when the abdominal muscles and accessory respiratory muscles are weak or deficient [3]. The patient in this case had received an abdominoplasty and did not show any significant loss of motor ability, so the appropriate amount of muscle relaxant was considered to be necessary for intubation. Hence, rocuronium $10 \mathrm{mg}(0.5 \mathrm{mg} / \mathrm{kg})$ was administered, and sevoflurane was used to maintain anesthesia. Due to the lack of muscle mass, the duration of muscle relaxants can be extended even when using the appropriate dosage in prune-belly patients. In addition, the duration can be extended by hypothermia or aminoglycoside antibiotics used in treating genitourinary infection; therefore, a minimal amount of muscle relaxant should be used.

During surgery for brachio-cephalic fistula formation, an anomaly was observed where the brachial artery was below the median nerve, but A-V fistula formation was performed. After A-V fistula formation, a permanent catheter was inserted in the subclavian vein, and the surgery was completed.

Within 10 minutes of suspending sevoflurane inhalation, spontaneous breathing was recovered with a tidal volume of $100-200 \mathrm{ml}$, a respiratory rate of $15-22$ beats/min and airway reflex; thus, extubation was performed, and the patient was moved to the recovery room. There were no significant abnormalities or dyspnea observed after extubation. Coughing was encouraged with chest physiotherapy without drugs. The patient showed stable vital signs in the recovery room and was transferred to a ward with no problems. In Prune-belly patients, due to a deficiency in the abdominal wall muscles and a flat diaphragm, there could be difficulty in effective coughing, and occasional chest wall deformities can worsen respiratory complications. As a result, there could be an increase in the risk of respiratory infection.

The patient started hemodialysis the next day using the permanent catheter, and there were no respiratory infections. The patient was discharged 1 week after surgery. Approximately 3 months later, a sufficient amount of blood flow at $250 \mathrm{ml} / \mathrm{min}$ in the A-V fistula was observed on the Doppler; thus, hemodialysis using the A-V fistula was possible.

Prune-belly syndrome is relatively rare, but in many cases, multiple abnormalities in different organs occur simultaneously. Each of the symptoms and diseases are all related to the anesthetic drug and method, so it is very important to have a clear understanding of this complex disease and related pathological physiology for the appropriate management of anesthesia. The anesthesiologist should perform a thorough preanesthetic evaluation for each patient according to the degree of the disease and type of malformation, carefully establishing the anesthesia plan, performing the anesthesia according to the plan, and continuing to carefully observe the patient after anesthesia to minimize complications before and after surgery in prune-belly patients.

\section{References}

1. King CR, Prescott G. Pathogenesis of the prune-belly anomalad. J Pediatr 1978; 93: 273-4.

2. Jennings RW. Prune belly syndrome. Semin Paediatr Surg 2000; 9: 115-20.

3. Holder JP. Pathophysiologic and anesthetic correlations of the prune-belly syndrome. AANA J 1989; 57: 137-41.

4. Straub E, Spranger J. Etiology and pathogenesis of the prune belly syndrome. Kidney Int 1981; 20: 695-9.

5. Kartz J, Benumof J, Kadis L. Anesthesia and uncommon diseases, pathophysiology and clinical correlations. 2nd ed. Philadelphia, W.B. Saunders Co. 1981, pp 146-50. 